\title{
Iterative Scheme with Errors for Common Zeros of Finite Accretive Mappings and Nonlinear Elliptic Systems
}

\author{
Li Wei and Ruilin Tan \\ School of Mathematics and Statistics, Hebei University of Economics and Business, Shijiazhuang 050061, China \\ Correspondence should be addressed to Li Wei; diandianba@yahoo.com
}

Received 6 January 2014; Accepted 9 February 2014; Published 3 April 2014

Academic Editor: Rudong Chen

Copyright ( 2014 L. Wei and R. Tan. This is an open access article distributed under the Creative Commons Attribution License, which permits unrestricted use, distribution, and reproduction in any medium, provided the original work is properly cited.

\begin{abstract}
We present a new iterative scheme with errors to solve the problems of finding common zeros of finite $m$-accretive mappings in a real Banach space. Strong convergence theorems are established, which extend the corresponding works given by some authors. Moreover, the relationship between zeros of $m$-accretive mappings and one kind of nonlinear elliptic systems is investigated, from which we can see that some restrictions imposed on the iterative scheme are valid and the solution of one kind of nonlinear elliptic systems can be approximated by a suitably defined iterative sequence.
\end{abstract}

\section{Introduction and Preliminaries}

Let $E$ be a real Banach space with norm $\|\cdot\|$ and let $E^{*}$ denote the dual space of $E$. We use " $\rightarrow$ " and " $\rightarrow$ " to denote strong and weak convergence, respectively. We denote the value of $f \in E^{*}$ at $x \in E$ by $\langle x, f\rangle$.

Let $C$ be a nonempty, closed, and convex subset of $E$ and let $Q$ be a mapping of $E$ onto $C$. Then $Q$ is said to be sunny [1] if $Q(Q(x)+t(x-Q(x)))=Q(x)$, for all $x \in E$ and $t \geq 0$.

A mapping $Q$ of $E$ into $E$ is said to be a retraction [1] if $Q^{2}=Q$. If a mapping $Q$ is a retraction, then $Q(z)=z$ for every $z \in R(Q)$, where $R(Q)$ is the range of $Q$.

A mapping $T: C \rightarrow C$ is said to be nonexpansive if $\|T x-T y\| \leq\|x-y\|$, for all $x, y \in C$. We use $F(T)$ to denote the fixed point set of $T$; that is, $F(T):=\{x \in C: T x=x\}$. A mapping $T: E \supset D(T) \rightarrow R(T) \subset E$ is said to be demiclosed at $p$ if whenever $\left\{x_{n}\right\}$ is a sequence in $D(T)$ such that $x_{n} \rightarrow$ $x \in D(T)$ and $T x_{n} \rightarrow p$, it follows that $T x=p$.

A subset $C$ of $E$ is said to be a sunny nonexpansive retract of $E$ [2] if there exists a sunny nonexpansive retraction of $E$ onto $C$ and it is called a nonexpansive retract of $E$ if there exists a nonexpansive retraction of $E$ onto $C$. If $E$ is reduced to a Hilbert space $H$, then the metric projection $P_{C}$ is a sunny nonexpansive retraction from $H$ to any closed and convex subset $C$ of $H$. But this is not true in a general Banach space. We note that if $E$ is smooth and $Q$ is a retraction of $C$ onto
$F(T)$, then $Q$ is sunny and nonexpansive if and only if for all $x \in C, z \in F(T),\langle Q x-x, J(Q x-z)\rangle \leq 0[3]$.

We use $J$ to denote the normalized duality mapping from $E$ to $2^{E^{*}}$ which is defined by

$$
J x:=\left\{f \in E^{*}:\langle x, f\rangle=\|x\|^{2}=\|f\|^{2}\right\}, \quad x \in E .
$$

It is well known that $J$ is single-valued if $E^{*}$ is strictly convex. Moreover, $J(c x)=c J x$, for all $x \in E$ and $c \in R^{1}$. We call that $J$ is weakly sequentially continuous if $\left\{x_{n}\right\}$ is a sequence in $E$ which converges weakly to $x$ it follows that $\left\{J x_{n}\right\}$ converges in weak* to $J x$.

A mapping $A: E \supset D(A) \rightarrow R(A) \subset E$ is called accretive if $\langle A x-A y, J(x-y)\rangle \geq 0$, for all $x, y \in D(A)$ and it is called $m$ accretive if $R(I+\lambda A)=E$, for all $\lambda>0$. Let $A^{-1} 0$ denote the set of zeros of $A$; that is, $A^{-1} 0:=\{x \in D(A): A x=0\}$. We denote by $J_{r}^{A}$ (for $\left.r>0\right)$ the resolvent of $A$; that is, $J_{r}^{A}:=(I+r A)^{-1}$. Then $J_{r}^{A}$ is nonexpansive and $F\left(J_{r}^{A}\right)=A^{-1} 0$.

Interest in accretive mappings, which is an important class of nonlinear operators, stems mainly from their firm connection with equations of evolution. It is well known that many physically significant problems can be modelled by initial value problems of the form

$$
x^{\prime}(t)+A x(t)=0, \quad x(0)=x_{0},
$$


where $A$ is an accretive mapping. Typical examples where such evolution equations occur can be found in the heat, wave, or Schrodinger equations. If $x(t)$ is dependent on $t$, then (2) is reduced to

$$
A u=0,
$$

whose solutions correspond to the equilibrium of the system (2). Consequently, within the past 40 years or so, considerable research efforts have been devoted to methods for finding approximate solutions of (3). An early fundamental result of accretive operators, due to Browder [4]. One classical method for studying the problem $0 \in A x$, where $A$ is an $m$-accretive mapping, is the following so-called proximal method (c.f. [5]):

$$
x_{0} \in H, \quad x_{n+1} \approx J_{r_{n}}^{A} x_{n}, \quad n \geq 0,
$$

where $J_{r_{n}}^{A}:=\left(I+r_{n} A\right)^{-1}$. It was shown that the sequence generated by (4) converges weakly or strongly to a zero point of $A$ under some conditions.

Recall that the following normal Mann iterative scheme to approximate the fixed point of a nonexpansive mapping $T: C \rightarrow C$ was introduced by Mann [6]:

$$
x_{0} \in C, \quad x_{n+1}=\left(1-\alpha_{n}\right) x_{n}+\alpha_{n} T x_{n}, \quad n \geq 0 .
$$

It was proved that, under some conditions, the sequence $\left\{x_{n}\right\}$ produced by (5) converges weakly to a point in $F(T)$.

Later, many mathematicians try to combine the ideas of proximal method and Mann iterative method to approximate the zeros of $m$-accretive mappings; see, for example, [7-14] and the references therein.

In particular, in 2007, Qin and Su [7] presented the following iterative scheme:

$$
\begin{aligned}
x_{1} & \in C, \\
y_{n} & =\beta_{n} x_{n}+\left(1-\beta_{n}\right) J_{r_{n}}^{A} x_{n}, \\
x_{n+1} & =\alpha_{n} u+\left(1-\alpha_{n}\right) y_{n} .
\end{aligned}
$$

And they showed that $\left\{x_{n}\right\}$ generated by the above scheme converges strongly to a zero of $A$.

Motivated by iterative schemes (4) and (5), Zegeye and Shahzad extended their discussion to the case of finite $m$ accretive mappings. They presented in [15] the following iterative scheme:

$$
x_{0} \in C, \quad x_{n+1}=\alpha_{n} u+\left(1-\alpha_{n}\right) S_{r} x_{n}, \quad n \geq 0,
$$

where $S_{r}=a_{0} I+a_{1} J_{A_{1}}+a_{2} J_{A_{2}}+\cdots+a_{l} J_{A_{l}}$ with $J_{A_{i}}=(I+$ $\left.A_{i}\right)^{-1}$ and $\sum_{i=0}^{l} a_{i}=1$. If $\bigcap_{i=1}^{l} A_{i}^{-1}(0) \neq \emptyset$, they proved that $\left\{x_{n}\right\}$ generated by $(7)$ converges strongly to the common zeros of $A_{i}(i=1,2, \ldots, l)$ under some conditions.

The work in [15] was then extended to the following one presented by $\mathrm{Hu}$ and Liu in [16]:

$$
x_{0} \in C, \quad x_{n+1}=\alpha_{n} u+\beta_{n} x_{n}+\vartheta_{n} S_{r_{n}} x_{n}, \quad n \geq 0,
$$

where $S_{r_{n}}=a_{0} I+a_{1} J_{r_{n}}^{A_{1}}+a_{2} J_{r_{n}}^{A_{2}}+\cdots+a_{l} J_{r_{n}}^{A_{l}}$ with $J_{r_{n}}^{A_{i}}=$ $\left(I+r_{n} A_{i}\right)^{-1}$ and $\sum_{i=0}^{l} a_{i}=1 .\left\{\alpha_{n}\right\},\left\{\beta_{n}\right\},\left\{\vartheta_{n}\right\} \subset(0,1)$, and $\alpha_{n}+\beta_{n}+\vartheta_{n}=1$. If $\bigcap_{i=1}^{l} A_{i}^{-1}(0) \neq \emptyset$, they proved that $\left\{x_{n}\right\}$ converges strongly to the common zeros of $A_{i}(i=1,2, \ldots, l)$ under some conditions.

In 2009, Yao et al. presented the following iterative scheme in the frame of Hilbert space in [17]:

$$
\begin{aligned}
x_{1} & \in C, \\
y_{n} & =P_{C}\left[\left(1-\alpha_{n}\right) x_{n}\right], \\
x_{n+1} & =\left(1-\beta_{n}\right) x_{n}+\beta_{n} T y_{n}, \quad n \geq 1,
\end{aligned}
$$

where $T: C \rightarrow C$ is a nonexpansive mapping with $F(T) \neq \emptyset$. Suppose $\left\{\alpha_{n}\right\}$ and $\left\{\beta_{n}\right\}$ are two real sequences in $(0,1)$ satisfying

(a) $\sum_{n=1}^{\infty} \alpha_{n}=+\infty$ and $\lim _{n \rightarrow \infty} \alpha_{n}=0$;

(b) $0<\liminf _{n \rightarrow \infty} \beta_{n} \leq \lim \sup _{n \rightarrow \infty} \beta_{n}<1$.

Then $\left\{x_{n}\right\}$ constructed by (9) converges strongly to a fixed point of $T$.

Motivated by the work in [15, 17], Shehu and Ezeora presented the following result in [2].

Theorem 1. Let $E$ be a real uniformly smooth and uniformly convex Banach space, and let $C$ be a nonempty, closed, and convex sunny nonexpansive retract of $E$, where $Q_{C}$ is the sunny nonexpansive retraction of $E$ onto $C$. Suppose the duality mapping $J: E \rightarrow E^{*}$ is weakly sequentially continuous. For each $i=1,2, \ldots, N$, let $A_{i}: C \rightarrow E$ be an $m$-accretive mapping such that $\bigcap_{i=1}^{N} A_{i}^{-1} 0 \neq \emptyset$. Let $\left\{\alpha_{n}\right\},\left\{\beta_{n}\right\} \subset(0,1)$ satisfy (a) and (b). Let $\left\{x_{n}\right\}$ be generated iteratively by

$$
\begin{aligned}
x_{1} & \in C, \\
y_{n} & =Q_{C}\left[\left(1-\alpha_{n}\right) x_{n}\right], \\
x_{n+1} & =\left(1-\beta_{n}\right) x_{n}+\beta_{n} S_{N} y_{n}, \quad n \geq 1,
\end{aligned}
$$

where $S_{N}:=a_{0} I+a_{1} J_{A_{1}}+a_{2} J_{A_{2}}+\cdots+a_{N} J_{A_{N}}$ with $J_{A_{i}}=$ $\left(I+A_{i}\right)^{-1}$, for $i=1,2, \ldots, N, 0<a_{k}<1$, for $k=$ $0,1,2, \ldots, N$, and $\sum_{k=0}^{N} a_{k}=1$. Then $\left\{x_{n}\right\}$ converges strongly to the common zero of $A_{i}$, where $i=1,2, \ldots, N$.

Inspired by the work in [2], we present the following iterative scheme with errors:

$$
\begin{aligned}
x_{1} & \in C, \\
u_{n} & =Q_{C}\left[\left(1-\alpha_{n}\right)\left(x_{n}+e_{n}\right)\right], \\
v_{n} & =\left(1-\beta_{n}\right) x_{n}+\beta_{n} S_{n} u_{n}, \\
x_{n+1} & =\gamma_{n} x_{n}+\left(1-\gamma_{n}\right) S_{n} v_{n}, \quad n \geq 1,
\end{aligned}
$$

where $\left\{e_{n}\right\} \subset E$ is the error sequence and $\left\{A_{i}\right\}_{i=1}^{N}$ is a finite family of $m$-accretive mappings. $S_{n}:=a_{0} I+a_{1} J_{r_{n, 1}}^{A_{1}}+a_{2} J_{r_{n, 2}}^{A_{2}}+$ $\cdots+a_{N} J_{r_{n, N}}^{A_{N}}, J_{r_{n, i}}^{A_{i}}=\left(I+r_{n, i} A_{i}\right)^{-1}$, for $i=1,2, \ldots, N ; \sum_{k=0}^{N} a_{k}=$ 
$1,0<a_{k}<1$, for $k=0,1,2, \ldots, N$. More details of iterative scheme (A) will be presented in Section 2. And, some strong convergence theorems are obtained.

Note that there are some differences between our work and Shehu and Ezeora's in [2] in the following aspects.

(i) $S_{n}$ in iterative scheme $(\mathrm{A})$ is different from $S_{N}$ in (10) since the former is changing with $n$ and the latter is not, which results in $A_{i}$ in scheme (A) having different coefficient $r_{n, i}$ for each different $i$.

(ii) The idea of three-step iteration is employed in our paper.

(iii) The error sequence $\left\{e_{n}\right\}$ is considered in the iterative scheme (A).

(iv) Recall that, in [2], Lemma 8 is a key tool to prove the convergence of $\left\{x_{n}\right\}$ generated by (10). In particular, to obtain the main result, they imposed an additional condition on the function $\beta$ in Lemma 8 that $\beta(t) \leq t / \max \left\{1,2 r_{1}\right\}$, where $r_{1}>0$ is a constant satisfying some conditions. One question arises: how to show the convergence of the iterative sequence $\left\{x_{n}\right\}$ if $\beta$ does not satisfy this additional condition? To answer the question, we will use Lemma 4 instead of Lemma 8.

In Section 3, we will discuss the relationship between zero point of finite $m$-accretive mappings and the solution of one kind of nonlinear elliptic systems involving $\left(p_{1}, p_{2}, \ldots, p_{N}\right)$ Laplacian operators. The discussion helps us not only to see that the topic of constructing iterative schemes to approximate zeros of $m$-accretive mappings is meaningful but also to see that the solution of $\left(p_{1}, p_{2}, \ldots, p_{N}\right)$-Laplacian elliptic systems can be obtained by an iterative scheme.

Next, we list some results we need in sequel.

Lemma 2 (see [18]). Let $E$ be a real uniformly convex Banach space, let $C$ be a nonempty, closed, and convex subset of $E$, and $T: C \rightarrow C$ is a nonexpansive mapping such that $F(T) \neq \emptyset$; then, $I-T$ is demiclosed at zero.

Lemma 3 (see [16]). Let $E$ be a strictly convex Banach space which has a uniformly Gâteaux differential norm, and let $C$ be a nonempty, closed, and convex subset of E. Let $\left\{A_{i}\right\}_{i=1}^{N}$ be a finite family of accretive mappings with $\bigcap_{i=1}^{N} A_{i}^{-1} 0 \neq \emptyset$, satisfying the following range conditions:

$$
\overline{D\left(A_{i}\right)} \subseteq C \subset \bigcap_{r>0} R\left(I+r A_{i}\right), \quad i=1,2, \ldots, N .
$$

Let $a_{0}, a_{1}, \ldots, a_{N}$ be real numbers in $(0,1)$ such that $\sum_{i=0}^{N} a_{i}=1$ and $S_{r_{n}}=a_{0} I+a_{1} J_{r_{n}}^{A_{1}}+a_{2} J_{r_{n}}^{A_{2}}+\cdots+a_{N} J_{r_{n}}^{A_{N}}$, where $J_{r_{n}}^{A_{i}}=\left(I+r_{n} A_{i}\right)^{-1}$ and $r_{n}>0$; then, $S_{r_{n}}$ is nonexpansive and $F\left(S_{r_{n}}\right)=\bigcap_{i=1}^{N} A_{i}^{-1} 0$.

Lemma 4 (see [13]). In a real Banach space E, the following inequality holds:

$$
\|x+y\|^{2} \leq\|x\|^{2}+2\langle y, j(x+y)\rangle, \quad \forall x, y \in E,
$$

where $j(x+y) \in J(x+y)$.
Lemma 5 (see [19]). Let $\left\{a_{n}\right\},\left\{b_{n}\right\}$, and $\left\{c_{n}\right\}$ be three sequences of nonnegative real numbers satisfying

$$
a_{n+1} \leq\left(1-c_{n}\right) a_{n}+b_{n} c_{n}, \quad \forall n \geq 1,
$$

where $\left\{c_{n}\right\} \subset(0,1)$ such that (i) $c_{n} \rightarrow 0$ and $\sum_{n=1}^{\infty} c_{n}=+\infty$ and (ii) either $\limsup _{n \rightarrow \infty} b_{n} \leq 0$ or $\sum_{n=1}^{\infty}\left|b_{n} c_{n}\right|<+\infty$. Then $\lim _{n \rightarrow \infty} a_{n}=0$.

Lemma 6 (see [20]). Let $\left\{x_{n}\right\}$ and $\left\{y_{n}\right\}$ be two bounded sequences in a Banach space $E$ such that $x_{n+1}=\beta_{n} x_{n}+$ $\left(1-\beta_{n}\right) y_{n}$, for $n \geq 1$. Suppose $\left\{\beta_{n}\right\} \subset(0,1)$ satisfying $0<\liminf _{n \rightarrow+\infty} \beta_{n} \leq \limsup _{n \rightarrow+\infty} \beta_{n}<1$. If $\lim \sup _{n \rightarrow+\infty}\left(\left\|y_{n+1}-y_{n}\right\|-\left\|x_{n+1}-x_{n}\right\|\right) \leq 0$, then $\lim _{n \rightarrow+\infty}\left\|y_{n}-x_{n}\right\|=0$.

Lemma 7 (see [21]). Let $E$ be a Banach space and let $A$ be an $m$-accretive mapping. For $\lambda>0, \mu>0$, and $x \in E$, one has

$$
J_{\lambda} x=J_{\mu}\left(\frac{\mu}{\lambda} x+\left(1-\frac{\mu}{\lambda}\right) J_{\lambda} x\right)
$$

where $J_{\lambda}=(I+\lambda A)^{-1}$ and $J_{\mu}=(I+\mu A)^{-1}$.

Lemma 8 (see [22]). Let $E$ be a real uniformly smooth Banach space; then there exists a nondecreasing continuous function $\beta$ : $[0,+\infty) \rightarrow[0,+\infty)$ with $\lim _{t \rightarrow 0^{+}} \beta(t)=0$ and $\beta(c t) \leq c \beta(t)$ for $c \geq 1$, such that, for all $x, y \in E$, the following inequality holds:

$$
\|x+y\|^{2} \leq\|x\|^{2}+2\langle y, J x\rangle+\max \{\|x\|, 1\}\|y\| \beta(\|y\|) .
$$

\section{Strong Convergence Theorem}

Lemma 9 (see [2]). Let $E$ be a real uniformly smooth and uniformly convex Banach space. Let $C$ be a nonempty, closed, and convex sunny nonexpansive retract of $E$, and let $Q_{C}$ be the sunny nonexpansive retraction of $E$ onto $C$. Let $T: C \rightarrow C$ be nonexpansive with $F(T) \neq \emptyset$. Suppose that the duality mapping $J: E \rightarrow E^{*}$ is weakly sequentially continuous. If for each $t \in(0,1)$ we define $T_{t}: C \rightarrow C$ by

$$
T_{t} x:=T Q_{C}[(1-t) x],
$$

then $T_{t}$ has a fixed point $z_{t}$, which is convergent strongly to the fixed point of $T$, as $t \rightarrow 0$. That is, $\lim _{t \rightarrow 0} z_{t}=z_{0} \in F(T)$.

Lemma 10. Let $E$ be a strictly convex Banach space and let $C$ be a nonempty, closed, and convex subset of E. Let $A_{i}: C \rightarrow$ $E(i=1,2, \ldots, N)$ be a finite family of $m$-accretive mappings such that $\bigcap_{i=1}^{N} A_{i}^{-1} 0 \neq \emptyset$.

Let $a_{0}, a_{1}, \ldots, a_{N}$ be real numbers in $(0,1)$ such that $\sum_{i=0}^{N} a_{i}=1$ and $S_{n}=a_{0} I+a_{1} J_{r_{n, 1}}^{A_{1}}+a_{2} J_{r_{n, 2}}^{A_{2}}+\cdots+a_{N} J_{r_{n, N}}^{A_{N}}$, where $J_{r_{n, i}}^{A_{i}}=\left(I+r_{n, i} A_{i}\right)^{-1}$ and $r_{n, i}>0$, for $i=1,2, \ldots, N$, and $n \geq 1$; then, $S_{n}: C \rightarrow C$ is nonexpansive and $F\left(S_{n}\right)=\bigcap_{i=1}^{N} A_{i}^{-1} 0$, for $n \geq 1$.

Proof. The main idea of the proof is essentially from that in [15] or Lemma 3. For the sake of completeness, we present the proof in the following. 
It is easy to check that $S_{n}: C \rightarrow C$ is nonexpansive and $\bigcap_{i=1}^{N} A_{i}^{-1} 0 \subset F\left(S_{n}\right)$.

On the other hand, for all $p \in F\left(S_{n}\right)$, then $p=S_{n} p=$ $a_{0} p+a_{1} J_{r_{n, 1}}^{A_{1}} p+a_{2} J_{r_{n, 2}}^{A_{2}} p+\cdots+a_{N} J_{r_{n, N}}^{A_{N}} p$.

For all $q \in \bigcap_{i=1}^{N} A_{i}^{-1} 0 \subset F\left(S_{n}\right)$, then

$$
\begin{aligned}
\|p-q\| & \leq a_{0}\|p-q\|+a_{1}\left\|J_{r_{n, 1}}^{A_{1}} p-q\right\|+\cdots+a_{N}\left\|J_{r_{n, N}}^{A_{N}} p-q\right\| \\
& \leq\left(a_{0}+a_{1}+\cdots+a_{N-1}\right)\|p-q\|+a_{N}\left\|J_{r_{n, N}}^{A_{N}} p-q\right\| \\
& =\left(1-a_{N}\right)\|p-q\|+a_{N}\left\|J_{r_{n, N}}^{A_{N}} p-q\right\| \leq\|p-q\| .
\end{aligned}
$$

Therefore, $\|p-q\|=\left(1-a_{N}\right)\|p-q\|+a_{N}\left\|J_{r_{n, N}}^{A_{N}} p-q\right\|$, which implies that $\|p-q\|=\left\|J_{r_{n, N}}^{A_{N}} p-q\right\|$. Similarly, $\|p-q\|=\| J_{r_{n, 1}}^{A_{1}} p-$ $q\|=\cdots=\| J_{r_{n, N}}^{A_{N}} p-q \|$.

Then $\|p-q\|=\|\left(a_{1} / \sum_{i=1}^{N} a_{i}\right)\left(J_{r_{n, 1}}^{A_{1}} p-q\right)+$ $\left(a_{2} / \sum_{i=1}^{N} a_{i}\right)\left(J_{r_{n, 2}}^{A_{2}} p-q\right)+\cdots+\left(a_{N} / \sum_{i=1}^{N} a_{i}\right)\left(J_{r_{n, N}}^{A_{N}} p-q\right) \|$, which implies from the strictly convexity of $E$ that $p-q=J_{r_{n, 1}}^{A_{1}} p-q=J_{r_{n, 2}}^{A_{2}} p-q=\cdots=J_{r_{n, N}}^{A_{N}} p-q$.

Therefore, $J_{r_{n, i}}^{A_{i}} p=p$, for $i=1,2, \ldots, N$. And then $p \in$ $\bigcap_{i=1}^{N} A_{i}^{-1} 0$, which completes the proof.

Lemma 11. Let $E, C$, and $S_{n}$ be the same as those in Lemma 10. Suppose $\bigcap_{i=1}^{N} A_{i}^{-1} 0 \neq \emptyset$. Then $S_{n}^{2}: C \rightarrow C$ is nonexpansive and $F\left(S_{n}^{2}\right)=\bigcap_{i=1}^{N} A_{i}^{-1} 0$.

Proof. From Lemma 10, we have $F\left(S_{n}\right)=\bigcap_{i=1}^{N} A_{i}^{-1} 0$. It is easy to check that $S_{n}^{2}: C \rightarrow C$ is nonexpansive. So, it suffices to show that $F\left(S_{n}^{2}\right) \subset F\left(S_{n}\right)$ since $F\left(S_{n}\right) \subset F\left(S_{n}^{2}\right)$ is trivial.

For all $p \in F\left(S_{n}^{2}\right)$, then $p=S_{n}^{2} p$.

For all $q \in F\left(S_{n}\right) \subset F\left(S_{n}^{2}\right)$, then $q=S_{n}^{2} q$. Now,

$$
\begin{aligned}
\|p-q\| & \leq\left\|S_{n} p-S_{n} q\right\|=\left\|S_{n} p-q\right\| \\
& \leq a_{0}\|p-q\|+a_{1}\left\|J_{r_{n, 1}}^{A_{1}} p-q\right\|+\cdots+a_{N}\left\|J_{r_{n, N}}^{A_{N}} p-q\right\| \\
& =\left(1-a_{N}\right)\|p-q\|+a_{N}\left\|J_{r_{n, N}}^{A_{N}} p-q\right\| \leq\|p-q\| .
\end{aligned}
$$

Therefore, $\|p-q\|=\left(1-a_{N}\right)\|p-q\|+a_{N}\left\|J_{r_{n, N}}^{A_{N}} p-q\right\|$, which implies that $\|p-q\|=\left\|J_{r_{n, N}}^{A_{N}} p-q\right\|$. Similarly, $\|p-q\|=\| J_{r_{n, 1}}^{A_{1}} p-$ $q\|=\cdots=\| J_{r_{n, N}}^{A_{N}} p-q \|$.

Then repeating the discussion in Lemma 10, we know that $J_{r_{n, i}}^{A_{i}} p=p$, for $i=1,2, \ldots, N$. And then $p \in F\left(S_{n}\right)$, which completes the proof.

Theorem 12. Let $E$ be a real uniformly smooth and uniformly convex Banach space. Let $C$ be a nonempty, closed, and convex sunny nonexpansive retract of $E$, where $Q_{C}$ is the sunny nonexpansive retraction of $E$ onto $C$. Let $A_{i}: C \rightarrow E$ be $m$-accretive mappings, where $i=1,2, \ldots, N$. Suppose that the duality mapping $: E \rightarrow E^{*}$ is weakly sequentially continuous and $D:=\bigcap_{i=1}^{N} A_{i}^{-1} 0 \neq \emptyset$. Let $\left\{x_{n}\right\}$ be generated by the iterative scheme $(A)$, where $S_{n}:=a_{0} I+a_{1} J_{r_{n, 1}}^{A_{1}}+a_{2} J_{r_{n, 2}}^{A_{2}}+\cdots+a_{N} J_{r_{n, N}}^{A_{N}}$, and $J_{r_{n, i}}^{A_{i}}=\left(I+r_{n, i} A_{i}\right)^{-1}$, for $i=1,2, \ldots, N, 0<a_{k}<1$, for $k=0,1,2, \ldots, N, \sum_{k=0}^{N} a_{k}=1$. Suppose that $\left\{e_{n}\right\} \subset E,\left\{\alpha_{n}\right\}$, $\left\{\beta_{n}\right\}$, and $\left\{\gamma_{n}\right\}$ are three sequences in $(0,1)$ and $\left\{r_{n, i}\right\} \subset(0,+\infty)$ satisfying the following conditions:

(i) $\alpha_{n} \rightarrow 0, \beta_{n} \rightarrow 0$, as $n \rightarrow \infty$;

(ii) $\sum_{n=1}^{\infty} \alpha_{n} \beta_{n}=+\infty$;

(iii) $0<\liminf _{n \rightarrow+\infty} \gamma_{n} \leq \lim \sup _{n \rightarrow+\infty} \gamma_{n}<1$;

(iv) $\sum_{n=1}^{\infty}\left|r_{n+1, i}-r_{n, i}\right|<+\infty$ and $r_{n, i} \geq \varepsilon>0$, for $n \geq 1$ and $i=1,2, \ldots, N$;

(v) $\left\|e_{n}\right\| / \alpha_{n} \rightarrow 0$, as $n \rightarrow+\infty$, and $\sum_{n=1}^{\infty}\left\|e_{n}\right\|<+\infty$.

Then $\left\{x_{n}\right\}$ converges strongly to a point $p_{0} \in D$.

Proof. We will split the proof into five steps.

Step 1. $\left\{x_{n}\right\},\left\{u_{n}\right\},\left\{S_{n} u_{n}\right\},\left\{v_{n}\right\}$, and $\left\{S_{n} x_{n}\right\}$ are all bounded.

We will first show that

$$
\forall p \in D, \quad\left\|x_{n+1}-p\right\| \leq M_{1}+\sum_{i=1}^{n}\left\|e_{i}\right\|,
$$

where $M_{1}=\max \left\{\left\|x_{1}-p\right\|,\|p\|\right\}$.

By using the induction method, we see that, for $n=1$, $\forall p \in D$,

$$
\begin{aligned}
\left\|x_{2}-p\right\| \leq & \gamma_{1}\left\|x_{1}-p\right\|+\left(1-\gamma_{1}\right)\left\|S_{1} v_{1}-p\right\| \\
\leq & \gamma_{1}\left\|x_{1}-p\right\|+\left(1-\gamma_{1}\right)\left\|v_{1}-p\right\| \\
\leq & \gamma_{1}\left\|x_{1}-p\right\|+\left(1-\gamma_{1}\right)\left(1-\beta_{1}\right)\left\|x_{1}-p\right\| \\
& +\beta_{1}\left(1-\gamma_{1}\right)\left\|u_{1}-p\right\| \\
\leq & \gamma_{1}\left\|x_{1}-p\right\|+\left(1-\gamma_{1}\right)\left(1-\beta_{1}\right)\left\|x_{1}-p\right\| \\
& +\beta_{1}\left(1-\gamma_{1}\right)\left\|\left(1-\alpha_{1}\right)\left(x_{1}+e_{1}\right)-p\right\| \\
\leq & {\left[1-\alpha_{1} \beta_{1}\left(1-\gamma_{1}\right)\right]\left\|x_{1}-p\right\|+\alpha_{1} \beta_{1}\left(1-\gamma_{1}\right)\|p\| } \\
& +\left(1-\alpha_{1}\right) \beta_{1}\left(1-\gamma_{1}\right)\left\|e_{1}\right\| \leq M_{1}+\left\|e_{1}\right\| .
\end{aligned}
$$

Suppose that (19) is true for $n=k$. Then, for $n=k+1$,

$$
\begin{aligned}
\left\|x_{k+2}-p\right\| \leq & \gamma_{k+1}\left\|x_{k+1}-p\right\|+\left(1-\gamma_{k+1}\right)\left\|v_{k+1}-p\right\| \\
\leq & \gamma_{k+1}\left\|x_{k+1}-p\right\|+\left(1-\gamma_{k+1}\right) \\
& \times\left[\left(1-\beta_{k+1}\right)\left\|x_{k+1}-p\right\|+\beta_{k+1}\left\|u_{k+1}-p\right\|\right]
\end{aligned}
$$




$$
\begin{aligned}
& \leq \gamma_{k+1}\left\|x_{k+1}-p\right\|+\left(1-\gamma_{k+1}\right) \\
& \quad \times\left[\left(1-\beta_{k+1}\right)\left\|x_{k+1}-p\right\|+\beta_{k+1}\right. \\
& \left.\quad \times\left\|\left(1-\alpha_{k+1}\right)\left(x_{k+1}+e_{k+1}\right)-p\right\|\right] \\
& \leq\left[1-\alpha_{k+1} \beta_{k+1}\left(1-\gamma_{k+1}\right)\right]\left\|x_{k+1}-p\right\| \\
& +\alpha_{k+1} \beta_{k+1}\left(1-\gamma_{k+1}\right)\|p\| \\
& +\beta_{k+1}\left(1-\alpha_{k+1}\right)\left(1-\gamma_{k+1}\right)\left\|e_{k+1}\right\| \\
& \leq M_{1}+\left[1-\alpha_{k+1} \beta_{k+1}\left(1-\gamma_{k+1}\right)\right] \\
& \quad \times \sum_{i=1}^{k}\left\|e_{i}\right\|+\left(1-\alpha_{k+1}\right) \beta_{k+1}\left(1-\gamma_{k+1}\right)\left\|e_{k+1}\right\| \\
& \leq M_{1}+\sum_{i=1}^{k+1}\left\|e_{i}\right\| .
\end{aligned}
$$

Thus (19) is true for all $n \in N$. Since $\sum_{n=1}^{\infty}\left\|e_{n}\right\|<+\infty$, then (19) ensures that $\left\{x_{n}\right\}$ is bounded.

For all $p \in D$, from $\left\|u_{n}-p\right\| \leq\left\|\left(1-\alpha_{n}\right)\left(x_{n}+e_{n}\right)-p\right\| \leq$ $\left\|x_{n}\right\|+\left\|e_{n}\right\|+\|p\|$, we see that $\left\{u_{n}\right\}$ is bounded.

Since $\left\|S_{n} u_{n}\right\| \leq\left\|S_{n} u_{n}-S_{n} p\right\|+\|p\| \leq\left\|u_{n}-p\right\|+\|p\|$, then $\left\{S_{n} u_{n}\right\}$ is bounded. Since both $\left\{S_{n} u_{n}\right\}$ and $\left\{x_{n}\right\}$ are bounded, then $\left\{v_{n}\right\}$ is bounded. Similarly, $\left\{S_{n} x_{n}\right\},\left\{J_{r_{n, i}}^{A_{i}} u_{n}\right\}$, and $\left\{J_{r_{n, i}}^{A_{i}} v_{n}\right\}$ are all bounded, for $i=1,2, \ldots, N$.

Then we set $M_{2}=\sup \left\{\left\|u_{n}\right\|,\left\|J_{r_{n, i}}^{A_{i}} u_{n}\right\|,\left\|v_{n}\right\|,\left\|J_{r_{n, i}}^{A_{i}} v_{n}\right\|: n \geq\right.$ $1, i=1,2, \ldots, N\}$.

Step 2. $\lim _{n \rightarrow \infty}\left\|x_{n}-S_{n} v_{n}\right\|=0$ and $\lim _{n \rightarrow \infty}\left\|x_{n+1}-x_{n}\right\|=0$.

In fact,

$$
\begin{aligned}
& \left\|S_{n+1} v_{n+1}-S_{n} v_{n}\right\| \\
& \quad \leq a_{0}\left\|v_{n+1}-v_{n}\right\|+\sum_{i=1}^{N} a_{i}\left\|J_{r_{n+1, i}}^{A_{i}} v_{n+1}-J_{r_{n, i}}^{A_{i}} v_{n}\right\| .
\end{aligned}
$$

Next, we discuss $\left\|J_{r_{n+1, i}}^{A_{i}} v_{n+1}-J_{r_{n, i}}^{A_{i}} v_{n}\right\|$.

If $r_{n, i} \leq r_{n+1, i}$, then, by using Lemma 7 , we have

$$
\begin{aligned}
\| J_{r_{n+1, i}}^{A_{i}} & v_{n+1}-J_{r_{n, i}}^{A_{i}} v_{n} \| \\
& =\left\|J_{r_{n, i}}^{A_{i}}\left(\frac{r_{n, i}}{r_{n+1, i}} v_{n+1}+\left(1-\frac{r_{n, i}}{r_{n+1, i}}\right) J_{r_{n+1, i}}^{A_{i}} v_{n+1}\right)-J_{r_{n, i}}^{A_{i}} v_{n}\right\| \\
& \leq\left\|\frac{r_{n, i}}{r_{n+1, i}} v_{n+1}+\left(1-\frac{r_{n, i}}{r_{n+1, i}}\right) J_{r_{n+1, i}}^{A_{i}} v_{n+1}-v_{n}\right\| \\
& \leq \frac{r_{n, i}}{r_{n+1, i}}\left\|v_{n+1}-v_{n}\right\|+\left(1-\frac{r_{n, i}}{r_{n+1, i}}\right)\left\|J_{r_{n+1, i}}^{A_{i}} v_{n+1}-v_{n}\right\| \\
& \leq\left\|v_{n+1}-v_{n}\right\|+\frac{r_{n+1, i}-r_{n, i}}{\varepsilon}\left\|J_{r_{n+1, i}}^{A_{i}} v_{n+1}-v_{n}\right\| .
\end{aligned}
$$

If $r_{n+1, i} \leq r_{n, i}$, then imitating the proof of (23), we have

$$
\begin{aligned}
& \left\|J_{r_{n+1, i}}^{A_{i}} v_{n+1}-J_{r_{n, i}}^{A_{i}} v_{n}\right\| \\
& \quad \leq\left\|v_{n+1}-v_{n}\right\|+\frac{r_{n, i}-r_{n+1, i}}{\varepsilon}\left\|J_{r_{n+1, i}}^{A_{i}} v_{n+1}-v_{n}\right\| .
\end{aligned}
$$

Combining (23) and (24), we have

$$
\begin{aligned}
& \left\|J_{r_{n+1, i}}^{A_{i}} v_{n+1}-J_{r_{n, i}}^{A_{i}} v_{n}\right\| \\
& \quad \leq\left\|v_{n+1}-v_{n}\right\|+\frac{\left|r_{n, i}-r_{n+1, i}\right|}{\varepsilon}\left\|J_{r_{n+1, i}}^{A_{i}} v_{n+1}-v_{n}\right\| \\
& \quad \leq\left\|v_{n+1}-v_{n}\right\|+\frac{2\left|r_{n, i}-r_{n+1, i}\right|}{\varepsilon} M_{2} .
\end{aligned}
$$

Putting (25) into (22), we have

$\left\|S_{n+1} v_{n+1}-S_{n} v_{n}\right\| \leq\left\|v_{n+1}-v_{n}\right\|+\frac{2 M_{2}}{\varepsilon} \sum_{i=1}^{N}\left|r_{n, i}-r_{n+1, i}\right|$.

Similarly, we have

$\left\|S_{n+1} u_{n+1}-S_{n} u_{n}\right\| \leq\left\|u_{n+1}-u_{n}\right\|+\frac{2 M_{2}}{\varepsilon} \sum_{i=1}^{N}\left|r_{n, i}-r_{n+1, i}\right|$.

Therefore, from (26) and (27), we have

$\left\|S_{n+1} v_{n+1}-S_{n} v_{n}\right\|$

$$
\begin{aligned}
\leq & \left\|v_{n+1}-v_{n}\right\|+\frac{2 M_{2}}{\varepsilon} \sum_{i=1}^{N}\left|r_{n, i}-r_{n+1, i}\right| \\
\leq & \left\|x_{n+1}-x_{n}\right\|+\beta_{n}\left\|x_{n}\right\|+\beta_{n+1}\left\|x_{n+1}\right\| \\
& +\left|\beta_{n+1}-\beta_{n}\right|\left\|S_{n+1} u_{n+1}\right\|+\beta_{n}\left\|S_{n+1} u_{n+1}-S_{n} u_{n}\right\| \\
& +\frac{2 M_{2}}{\varepsilon} \sum_{i=1}^{N}\left|r_{n, i}-r_{n+1, i}\right| \\
\leq & \left\|x_{n+1}-x_{n}\right\|+\beta_{n}\left\|x_{n}\right\|+\beta_{n+1}\left\|x_{n+1}\right\| \\
& +\left|\beta_{n+1}-\beta_{n}\right|\left\|S_{n+1} u_{n+1}\right\|+\beta_{n}\left\|u_{n+1}-u_{n}\right\| \\
& +\frac{4 M_{2}}{\varepsilon} \sum_{i=1}^{N}\left|r_{n, i}-r_{n+1, i}\right| \\
\leq & \left\|x_{n+1}-x_{n}\right\|+\beta_{n}\left\|x_{n}\right\|+\beta_{n+1}\left\|x_{n+1}\right\| \\
& +\left|\beta_{n+1}-\beta_{n}\right|\left\|S_{n+1} u_{n+1}\right\| \\
& +\beta_{n}\left\|\left(1-\alpha_{n+1}\right)\left(x_{n+1}+e_{n+1}\right)-\left(1-\alpha_{n}\right)\left(x_{n}+e_{n}\right)\right\| \\
& +\frac{4 M_{2}}{\varepsilon} \sum_{i=1}^{N}\left|r_{n, i}-r_{n+1, i}\right|
\end{aligned}
$$




$$
\begin{aligned}
& \leq\left(1+\beta_{n}\right)\left\|x_{n+1}-x_{n}\right\|+\left(\beta_{n}+\alpha_{n} \beta_{n}\right)\left\|x_{n}\right\| \\
& +\left(\beta_{n+1}+\alpha_{n+1} \beta_{n}\right)\left\|x_{n+1}\right\|+\left|\beta_{n+1}-\beta_{n}\right|\left\|S_{n+1} u_{n+1}\right\| \\
& +\beta_{n}\left\|e_{n+1}-e_{n}\right\|+\beta_{n}\left\|\alpha_{n+1} e_{n+1}-\alpha_{n} e_{n}\right\| \\
& +\frac{4 M_{2}}{\varepsilon} \sum_{i=1}^{N}\left|r_{n, i}-r_{n+1, i}\right|
\end{aligned}
$$

From Step 1, we know that $\limsup _{n \rightarrow+\infty}\left(\| S_{n+1} v_{n+1}-\right.$ $\left.S_{n} v_{n}\|-\| x_{n+1}-x_{n} \|\right) \leq 0$. Using Lemma 6, we have from (28) that $\lim _{n \rightarrow \infty}\left\|x_{n}-S_{n} v_{n}\right\|=0$ and then $\lim _{n \rightarrow \infty}\left\|x_{n+1}-x_{n}\right\|=$ $\lim _{n \rightarrow \infty}\left(1-\gamma_{n}\right)\left\|S_{n} v_{n}-x_{n}\right\|=0$.

Step 3. $\lim _{n \rightarrow \infty}\left\|x_{n}-S_{n} x_{n}\right\|=0$ and $\lim _{n \rightarrow \infty}\left\|x_{n}-S_{n}^{2} x_{n}\right\|=0$. In fact,

$$
\begin{aligned}
\left\|x_{n}-S_{n} x_{n}\right\| & \leq\left\|x_{n}-S_{n} v_{n}\right\|+\left\|S_{n} v_{n}-S_{n} x_{n}\right\| \\
& \leq\left\|x_{n}-S_{n} v_{n}\right\|+\left\|v_{n}-x_{n}\right\| \\
& \leq\left\|x_{n}-S_{n} v_{n}\right\|+\beta_{n}\left\|x_{n}-S_{n} u_{n}\right\| .
\end{aligned}
$$

Noticing the results of Steps 1 and 2 and $\beta_{n} \rightarrow 0$, we have $\lim _{n \rightarrow \infty}\left\|x_{n}-S_{n} x_{n}\right\|=0$.

Since $\left\|x_{n}-S_{n}^{2} x_{n}\right\| \leq\left\|x_{n}-S_{n} x_{n}\right\|+\left\|S_{n} x_{n}-S_{n}^{2} x_{n}\right\| \leq 2 \| x_{n}-$ $S_{n} x_{n} \|$, then $\left\|x_{n}-S_{n}^{2} x_{n}\right\| \rightarrow 0$, as $n \rightarrow \infty$.

Step 4. $\lim \sup _{n \rightarrow+\infty}\left\langle p_{0}, J\left(p_{0}-x_{n}\right)\right\rangle \leq 0$, where $p_{0}$ is an element in $D$.

From Lemma 11, we know that $S_{n}^{2}: C \rightarrow C$ is nonexpansive and $F\left(S_{n}^{2}\right)=D$. Then Lemma 9 implies that there exists $z_{t} \in C$ such that $z_{t}=S_{n}^{2} Q_{C}\left[(1-t) z_{t}\right]$ for $t \in(0,1)$. Moreover, $z_{t} \rightarrow p_{0} \in D$, as $t \rightarrow 0$.

Since $\left\|z_{t}-p_{0}\right\| \leq\left\|(1-t) z_{t}-p_{0}\right\| \leq(1-t)\left\|z_{t}-p_{0}\right\|+\left\|p_{0}\right\|$, then $\left\{z_{t}\right\}$ is bounded. Let $M_{3}=\sup \left\{\left\|z_{t}-x_{n}\right\|: n \geq 1, t>\right.$ $0\}$. Then from Step 1, we know that $M_{3}$ is a positive constant. Using Lemma 4, we have

$$
\begin{aligned}
\| z_{t} & -x_{n} \|^{2} \\
& =\left\|z_{t}-S_{n}^{2} x_{n}+S_{n}^{2} x_{n}-x_{n}\right\|^{2} \\
\leq & \left\|z_{t}-S_{n}^{2} x_{n}\right\|^{2}+2\left\langle S_{n}^{2} x_{n}-x_{n}, J\left(z_{t}-x_{n}\right)\right\rangle \\
\leq & \left\|z_{t}-S_{n}^{2} x_{n}\right\|^{2}+2\left\|S_{n}^{2} x_{n}-x_{n}\right\|\left\|z_{t}-x_{n}\right\| \\
\leq & \left\|(1-t) z_{t}-x_{n}\right\|^{2}+2\left\|S_{n}^{2} x_{n}-x_{n}\right\|\left\|z_{t}-x_{n}\right\| \\
\leq & \left\|z_{t}-x_{n}\right\|^{2}-2 t\left\langle z_{t}, J\left[(1-t) z_{t}-x_{n}\right]\right\rangle \\
& +2 M_{3}\left\|S_{n}^{2} x_{n}-x_{n}\right\| .
\end{aligned}
$$

So $\left\langle z_{t}, J\left[(1-t) z_{t}-x_{n}\right]\right\rangle \leq\left(M_{3} / t\right)\left\|S_{n}^{2} x_{n}-x_{n}\right\|$, which implies that $\lim _{t \rightarrow 0} \limsup _{n \rightarrow+\infty}\left\langle z_{t}, J\left[(1-t) z_{t}-x_{n}\right]\right\rangle \leq 0$ in view of Step 3.

Since $\left\{x_{n}\right\}$ is bounded and $J$ is uniformly continuous on each bounded subset of $E$, then $\left\langle p_{0}, J\left(p_{0}-x_{n}\right)-J\left[(1-t) z_{t}-\right.\right.$ $\left.\left.x_{n}\right]\right\rangle \rightarrow 0$, as $t \rightarrow 0$.
Moreover, noticing the fact that

$$
\begin{aligned}
\left\langle p_{0}, J\right. & \left.\left(p_{0}-x_{n}\right)\right\rangle \\
= & \left\langle p_{0}, J\left(p_{0}-x_{n}\right)-J\left[(1-t) z_{t}-x_{n}\right]\right\rangle \\
& +\left\langle p_{0}-z_{t}, J\left[(1-t) z_{t}-x_{n}\right]\right\rangle \\
& +\left\langle z_{t}, J\left[(1-t) z_{t}-x_{n}\right]\right\rangle,
\end{aligned}
$$

we have $\lim \sup _{n \rightarrow+\infty}\left\langle p_{0}, J\left(p_{0}-x_{n}\right)\right\rangle \leq 0$.

Since $\left\langle p_{0}, J\left[p_{0}-x_{n}-\left(1-\alpha_{n}\right) e_{n}+\alpha_{n} x_{n}\right]\right\rangle=\left\langle p_{0}, J\left[p_{0}-\right.\right.$ $\left.\left.\left.\left.x_{n}-\left(1-\alpha_{n}\right) e_{n}+\alpha_{n} x_{n}\right]-J\left(p_{0}-x_{n}\right)\right)\right\rangle+\left\langle p_{0}, J\left(p_{0}-x_{n}\right)\right)\right\rangle$ and $J$ is uniformly continuous on each bounded subset of $E$, then

$$
\limsup _{n \rightarrow+\infty}\left\langle p_{0}, J\left[p_{0}-x_{n}-\left(1-\alpha_{n}\right) e_{n}+\alpha_{n} x_{n}\right]\right\rangle \leq 0 .
$$

Step 5. $x_{n} \rightarrow p_{0}$, as $n \rightarrow+\infty$, where $p_{0} \in D$ is the same as that in Step 4.

Let $M_{4}=\sup \left\{\left\|\left(1-\alpha_{n}\right)\left(x_{n}+e_{n}\right)-p_{0}\right\|: n \geq 1\right\}$. By using Lemma 4 again, we have

$$
\begin{aligned}
\| x_{n+1} & -p_{0} \|^{2} \\
\leq & \gamma_{n}\left\|x_{n}-p_{0}\right\|^{2}+\left(1-\gamma_{n}\right)\left\|v_{n}-p_{0}\right\|^{2} \\
\leq & \gamma_{n}\left\|x_{n}-p_{0}\right\|^{2}+\left(1-\gamma_{n}\right)\left(1-\beta_{n}\right)\left\|x_{n}-p_{0}\right\|^{2} \\
& +\left(1-\gamma_{n}\right) \beta_{n}\left\|u_{n}-p_{0}\right\|^{2} \\
= & \left(1-\beta_{n}+\beta_{n} \gamma_{n}\right)\left\|x_{n}-p_{0}\right\|^{2}+\left(1-\gamma_{n}\right) \beta_{n}\left\|u_{n}-p_{0}\right\|^{2} \\
\leq & \left(1-\beta_{n}+\beta_{n} \gamma_{n}\right)\left\|x_{n}-p_{0}\right\|^{2} \\
& +\left(1-\gamma_{n}\right) \beta_{n}\left\|\left(1-\alpha_{n}\right)\left(x_{n}+e_{n}\right)-p_{0}\right\|^{2} \\
\leq & \left(1-\beta_{n}+\beta_{n} \gamma_{n}\right)\left\|x_{n}-p_{0}\right\|^{2}+\left(1-\gamma_{n}\right) \beta_{n}\left(1-\alpha_{n}\right) \\
& \times\left\|x_{n}-p_{0}\right\|^{2}+2\left(1-\gamma_{n}\right) \beta_{n}\left(1-\alpha_{n}\right) \\
& \times\left\langle e_{n}, J\left[\left(1-\alpha_{n}\right)\left(x_{n}+e_{n}\right)-p_{0}\right]\right\rangle+2 \alpha_{n} \beta_{n}\left(1-\gamma_{n}\right) \\
& \times\left\langle p_{0}, J\left[p_{0}-x_{n}-\left(1-\alpha_{n}\right) e_{n}+\alpha_{n} x_{n}\right]\right\rangle \\
\leq & {\left[\left(1-\alpha_{n} \beta_{n}\left(1-\gamma_{n}\right)\right]\left\|x_{n}-p_{0}\right\|^{2}+2\left(1-\gamma_{n}\right)\right.} \\
& \times\left(1-\alpha_{n}\right) \beta_{n} M_{4}\left\|e_{n}\right\|+2 \alpha_{n} \beta_{n}\left(1-\gamma_{n}\right) \\
& \times\left\langle p_{0}, J\left[p_{0}-x_{n}-\left(1-\alpha_{n}\right) e_{n}+\alpha_{n} x_{n}\right]\right\rangle .
\end{aligned}
$$

Let $c_{n}=\left(1-\gamma_{n}\right) \alpha_{n} \beta_{n}$; then (33) reduces to $\left\|x_{n+1}-p_{0}\right\|^{2} \leq$ $\left(1-c_{n}\right)\left\|x_{n}-p_{0}\right\|^{2}+2 c_{n}\left\{\left\langle p_{0}, J\left[p_{0}-x_{n}-\left(1-\alpha_{n}\right) e_{n}+\alpha_{n} x_{n}\right]\right\rangle+\right.$ $\left.\left(1-\alpha_{n}\right) M_{4}\left(\left\|e_{n}\right\| / \alpha_{n}\right)\right\}$.

From (32), (33), and the assumptions, by using Lemma 5, we know that $x_{n} \rightarrow p_{0}$, as $n \rightarrow+\infty$.

This completes the proof.

If, in Theorem 12, $C=E$, then we have the following theorem. 
Theorem 13. Let $E$ and $D$ be the same as those in Theorem 12. Suppose that the duality mapping $J: E \rightarrow E^{*}$ is weakly sequentially continuous. Let $A_{i}: E \rightarrow E(i=1,2, \ldots, N)$ be a finite family of $m$-accretive mappings. Let $\left\{e_{n}\right\} \subset E,\left\{\alpha_{n}\right\}$, $\left\{\beta_{n}\right\},\left\{\gamma_{n}\right\} \subset(0,1)$, and $\left\{r_{n, i}\right\} \subset(0,+\infty)$ satisfy the conditions presented in Theorem 12 .

Let $\left\{x_{n}\right\}$ be generated by the following scheme:

$$
\begin{aligned}
x_{1} & \in E, \\
u_{n} & =\left(1-\alpha_{n}\right)\left(x_{n}+e_{n}\right), \\
v_{n} & =\left(1-\beta_{n}\right) x_{n}+\beta_{n} S_{n} u_{n}, \\
x_{n+1} & =\gamma_{n} x_{n}+\left(1-\gamma_{n}\right) S_{n} v_{n}, \quad n \geq 1 .
\end{aligned}
$$

Then $\left\{x_{n}\right\}$ converges strongly to a point $p_{0} \in D$, where $S_{n}$ is the same as that in Theorem 12.

\section{Nonlinear Elliptic Systems with $\left(p_{1}, p_{2}, \ldots, p_{N}\right)$-Laplacian}

In what follows in this paper, unless otherwise stated, we will assume that $(2 N / N+1)<p_{i}<+\infty,\left(1 / p_{i}\right)+\left(1 / p_{i}^{\prime}\right)=1$. We use $\|\cdot\|_{p_{i}},\|\cdot\|_{p_{i}^{\prime}}$, and $\|\cdot\|_{1, p_{i}, \Omega}$ to denote the norms of spaces $L^{p_{i}}(\Omega), L^{p_{i}^{\prime}}(\Omega)$, and $W^{1, p_{i}}(\Omega)$, respectively, where $i=$ $1,2, \ldots, N$.

Now, we will examine the following nonlinear elliptic systems:

$$
\begin{aligned}
& -\operatorname{div}\left[\left(C_{i}(x)+|\nabla u|^{2}\right)^{\left(p_{i}-2\right) / 2} \nabla u\right]=f_{i}(x), \quad \text { a.e. } x \in \Omega, \\
& -\left\langle\vartheta,\left(C_{i}(x)+|\nabla u|^{2}\right)^{\left(p_{i}-2\right) / 2} \nabla u\right\rangle=0, \quad \text { a.e. } x \in \Gamma, \\
& i=1,2, \ldots, N \text {. }
\end{aligned}
$$

In (C), $\Omega$ is a bounded conical domain of a Euclidean space $R^{N}$ with its boundary $\Gamma \in C^{1}$, (c.f. [23]). $f_{i}(x) \in L^{p_{i}^{\prime}}(\Omega)$ is a given function and $0 \leq C_{i}(x) \in L^{p_{i}}(\Omega)$, for $i=1,2, \ldots, N$. $\vartheta$ denotes the exterior normal derivative of $\Gamma$.

Lemma 14 (see [24]). Define the mapping $B_{p_{i}}: W^{1, p_{i}}(\Omega) \rightarrow$ $\left(W^{1, p_{i}}(\Omega)\right)^{*}$ by

$$
\left(v, B_{p_{i}} u\right)=\int_{\Omega}\left\langle\left(C_{i}(x)+|\nabla u|^{2}\right)^{\left(p_{i}-2\right) / 2} \nabla u, \nabla v\right\rangle d x
$$

for any $u, v \in W^{1, p_{i}}(\Omega)$. Then, $B_{p_{i}}$ is everywhere defined, strictly monotone, hemicontinuous, coercive, and maximal monotone, for $i=1,2, \ldots, N$.

Lemma 15 (see [24]). Define the mapping $A_{i}: L^{2}(\Omega) \rightarrow$ $L^{2}(\Omega)$ by

$$
\begin{gathered}
D\left(A_{i}\right)=\left\{u(x) \in L^{2}(\Omega): \text { there exists } g_{i}(x) \in L^{2}(\Omega)\right. \\
\text { such that } \left.g_{i}(x)=B_{p_{i}} u\right\}
\end{gathered}
$$

for any $u \in D\left(A_{i}\right), A_{i} u=g_{i}(x)$. Then, $A_{i}$ is $m$-accretive, for $i=1,2, \ldots, N$.

Lemma 16 (see [24]). For $i=1,2, \ldots, N$, define the mapping $A_{p_{i}}: L^{p_{i}}(\Omega) \rightarrow L^{p_{i}}(\Omega)$ in the following way:

(i) if $p_{i} \geq 2, D\left(A_{p_{i}}\right)=\left\{u(x) \in L^{p_{i}}(\Omega)\right.$ : there exists $h_{i}(x) \in L^{p_{i}}(\Omega)$ such that $\left.h_{i}(x)=B_{p_{i}} u\right\}$, then for any $u \in D\left(A_{p_{i}}\right), A_{p_{i}} u=h_{i}(x)$,

(ii) if $2 N /(N+1)<p_{i}<2$, we define $A_{p_{i}}: L^{p_{i}}(\Omega) \rightarrow$ $L^{p_{i}}(\Omega)$ as the $L^{p_{i}}$-closure of $A_{i}: L^{2}(\Omega) \rightarrow L^{2}(\Omega)$ defined in (i) above.

Then, $A_{p_{i}}$ is $m$-accretive, for $i=1,2, \ldots, N$.

Lemma 17 (see [25]). Let $\Omega$ be a bounded conical domain in $R^{N}$. If $m p>N$, then $W^{m, p}(\Omega) \hookrightarrow \hookrightarrow C_{B}(\Omega)$; if $0<m p \leq N$ and $q_{0}=N p /(N-m p)$, then $W^{m, p}(\Omega) \hookrightarrow \hookrightarrow L^{q}(\Omega)$, where $1 \leq q<q_{0}$.

Lemma 18 (see [26]). Let $X_{0}$ denote the closed subspace of all constant functions in $W^{1, p}(\Omega)$. Let $X$ be the quotient space $W^{1, p}(\Omega) / X_{0}$. For $u \in W^{1, p}(\Omega)$, define the mapping $P$ : $W^{1, p}(\Omega) \rightarrow X_{0}$ by $P u=(1 /$ meas $(\Omega)) \int_{\Omega} u d x$. Then, there is a constant $C>0$, such that $\forall u \in W^{1, p}(\Omega)$,

$$
\|u-P u\|_{p} \leq C\|\nabla u\|_{\left(L^{p}(\Omega)\right)^{N}}
$$

Theorem 19. For $i=1,2, \ldots, N$, one has $A_{p_{i}}^{-1} 0=\{u \in$ $L^{p_{i}}(\Omega): u(x) \equiv$ Constant on $\left.\Omega\right\}$.

Proof. (i) $p_{i} \geq 2$.

Let $u(x) \in A_{p_{i}}^{-1} 0$; then $0=$ $\left(u, B_{p_{i}} u\right)=\int_{\Omega}\left(C_{i}(x)+|\nabla u|^{2}\right)^{\left(p_{i}-2\right) / 2}|\nabla u|^{2} d x \geq \int_{\Omega}|\nabla u|^{p_{i}} d x \geq$ 0 , which implies that $u(x) \equiv$ Constant. That is, $A_{p_{i}}^{-1} 0 \subset\left\{u \in L^{p_{i}}(\Omega): u(x) \equiv\right.$ Constant $\}$.

On the other hand, suppose $u(x) \equiv$ Constant. Then $0=$ $\left(v, B_{p_{i}} u\right)$, for all $v \in W^{1, p_{i}}(\Omega)$. Then $u \in A_{p_{i}}^{-1} 0$. Therefore, the result holds.

(ii) $2 N /(N+1)<p_{i}<2$.

Suppose $u \in L^{p_{i}}(\Omega)$ and $u(x) \equiv$ Constant. Then let $u_{n} \equiv$ $u$, and we can easily see that $u \in A_{p_{i}}^{-1} 0$ in view of the definition of $A_{p_{i}}$.

On the other hand, let $u \in A_{p_{i}}^{-1} 0$. Then there exist $\left\{u_{n}\right\}$ and $\left\{f_{n}\right\}$ in $L^{2}(\Omega)$ such that $u_{n} \rightarrow u, f_{n} \rightarrow 0$ in $L^{p_{i}}(\Omega)$ and $A_{i} u_{n}=f_{n}$. Now, define the following functions:

$$
\begin{gathered}
\chi(t)= \begin{cases}|t|^{p_{i}-1} \text { sgnt, } & \text { if }|t| \geq 1 \\
t, & \text { if }|t|<1,\end{cases} \\
\xi(t)= \begin{cases}|t|^{2-\left(2 / p_{i}\right)} \text { sgnt, } & \text { if }|t| \geq 1 \\
t, & \text { if }|t|<1 .\end{cases}
\end{gathered}
$$

Then for $u \in L^{2}(\Omega)$, the function $t \in R \rightarrow \int_{\Omega} \xi(u+$ $t) d x \in R$ is continuous and $\lim _{t \rightarrow \pm \infty} \int_{\Omega} \xi(u+t) d x= \pm \infty$. Therefore, there exists $t_{u} \in R$ such that $\int_{\Omega} \xi\left(u+t_{u}\right) d x=0$. 
So, for $u_{n} \in L^{2}(\Omega)$, we may assume that there exist $t_{n} \in R$ such that $\int_{\Omega} \xi\left(u_{n}+t_{n}\right) d x=0$ and $A_{i} u_{n}=f_{n}$, for $n \geq 1$. Let $v_{n}=u_{n}+t_{n}$; then $A_{i} v_{n}=A_{i} u_{n}=f_{n}$, for $n \geq 1$.

Now, compute the following:

$$
\begin{aligned}
& \left\|f_{n}\right\|_{p_{i}}\left(\int_{\left|v_{n}\right| \leq 1}\left|v_{n}\right|^{p_{i}^{\prime}} d x+\int_{\left|v_{n}\right| \geq 1}\left|v_{n}\right|^{2} d x\right)^{\left(p_{i}-1\right) / p_{i}} \\
& \quad \geq\left\|f_{n}\right\|_{p_{i}}\left(\int_{\left|v_{n}\right| \leq 1}\left|v_{n}\right|^{p_{i}^{\prime}} d x+\int_{\left|v_{n}\right| \geq 1}\left|v_{n}\right|^{p_{i}} d x\right)^{\left(p_{i}-1\right) / p_{i}} \\
& \quad \leq\left\|f_{n}\right\|_{p_{i}}\left\|\chi\left(v_{n}\right)\right\|_{p_{i^{\prime}}} \geq\left(\chi\left(v_{n}\right), f_{n}\right) \\
& \quad \geq\left(\chi\left(v_{n}\right), A_{i} v_{n}\right) \geq \int_{\Omega}\left|\nabla v_{n}\right|^{p_{i}} \chi^{\prime}\left(v_{n}\right) d x \\
& \geq \text { Const. } \int_{\Omega}\left|\nabla\left(\xi\left(v_{n}\right)\right)\right|^{p_{i}} d x .
\end{aligned}
$$

Using Lemma 18,

$$
\int_{\Omega}\left|\nabla\left(\xi\left(v_{n}\right)\right)\right|^{p_{i}} d x \geq \text { Const. }\left\|\xi\left(v_{n}\right)\right\|_{1, p_{i}, \Omega}^{p_{i}} .
$$

Then Lemma 17 implies that

$$
\begin{aligned}
& \left\|\xi\left(v_{n}\right)\right\|_{1, p_{i}, \Omega}^{p_{i}} \\
& \quad \geq \text { Const. }\left\|\xi\left(v_{n}\right)\right\|_{p_{i}^{\prime}}^{p_{i}} \\
& \quad=\text { Const. }\left(\int_{\left|v_{n}\right| \leq 1}\left|v_{n}\right|^{p_{i}^{\prime}} d x+\int_{\left|v_{n}\right| \geq 1}\left|v_{n}\right|^{\left(2-\left(2 / p_{i}\right)\right) p_{i}^{\prime}} d x\right)^{p_{i} / p_{i}^{\prime}} \\
& =\text { Const. }\left(\int_{\left|v_{n}\right| \leq 1}\left|v_{n}\right|^{p_{i}^{\prime}} d x+\int_{\left|v_{n}\right| \geq 1}\left|v_{n}\right|^{2} d x\right)^{p_{i} / p_{i}^{\prime}} .
\end{aligned}
$$

From (38), (39), and (40), we know that $\left\|\xi\left(v_{n}\right)\right\|_{1, p_{i}, \Omega}^{p_{i}} \leq$ Const. $\left\|f_{n}\right\|_{p_{i}} \rightarrow 0$, as $n \rightarrow \infty$. Then $\xi\left(v_{n}\right) \rightarrow 0$ in $L^{p_{i}^{\prime}}(\Omega)$. Since Nemytskyi mapping $u \in L^{p_{i}^{\prime}}(\Omega) \rightarrow \xi^{-1}(u) \in L^{p_{i}}(\Omega)$ is continuous, then $v_{n} \rightarrow 0$ in $L^{p_{i}}(\Omega)$. And then $u(x) \equiv$ Constant.

This completes the proof.

Remark 20. Theorem 19 helps us to see the assumption that $\bigcap_{i=1}^{N} A_{p_{i}}^{-1} 0 \neq \emptyset$ in Theorems 12 and 13 are valid.

Remark 21. If we set $C_{p_{i}}=A_{p_{i}}-f_{i}(x)$, then $C_{p_{i}}$ is also $m$-accretive. And then iterative scheme (A) can be used to approximate the element in $\bigcap_{i=1}^{N} C_{p_{i}}^{-1} 0$, which is just the solution of nonlinear elliptic system $(\mathrm{C})$.

Remark 22. If $C_{i}(x) \equiv 0$, then (C) is reduced to the following nonlinear elliptic systems with $\left(p_{1}, p_{2}, \ldots, p_{N}\right)$-Laplacian:

$$
\begin{array}{r}
-\Delta_{p_{i}} u=f_{i}(x), \quad \text { a.e. } x \in \Omega, \\
-\left\langle\vartheta,|\nabla u|^{p_{i}-2} \nabla u\right\rangle=0, \quad \text { a.e. } x \in \Gamma, \\
i=1,2, \ldots, N .
\end{array}
$$

Moreover, the solution of (D) is the zero of finitely many suitably defined $m$-accretive mappings, which can be approximated by iterative scheme (A).

\section{Conflict of Interests}

The authors declare that there is no conflict of interests regarding the publication of this paper.

\section{Acknowledgments}

This paper is supported by the National Natural Science Foundation of China (no. 11071053), the Natural Science Foundation of Hebei Province (no. A2014207010), the Key Project of Science and Research of Hebei Educational Department (ZH2012080), and the Key Project of Science and Research of Hebei University of Economics and Business (2013KYZ01).

\section{References}

[1] W. Takahashi, "Proximal point algorithms and four resolvents of nonlinear operators of monotone type in Banach spaces," Taiwanese Journal of Mathematics, vol. 12, no. 8, pp. 1883-1910, 2008.

[2] Y. Shehu and J. N. Ezeora, "Path convergence and approximation of common zeroes of a finite family of $m$-accretive mappings in Banach spaces," Abstract and Applied Analysis, vol. 2010, Article ID 285376, 14 pages, 2010.

[3] W. Takahashi, Nonlinear Functional Analysis, Fixed Point Theory and Its Application, Yokohama Publishers, Yokohama, Japan, 2000.

[4] F. E. Browder, "Nonlinear mappings of nonexpansive and accretive type in Banach spaces," Bulletin of the American Mathematical Society, vol. 73, pp. 875-882, 1967.

[5] R. T. Rockafellar, "Monotone operators and the proximal point algorithm," SIAM Journal on Control and Optimization, vol. 14, no. 5, pp. 877-898, 1976.

[6] W. R. Mann, "Mean value methods in iteration," Proceedings of the American Mathematical Society, vol. 4, pp. 506-510, 1953.

[7] X. Qin and Y. Su, "Approximation of a zero point of accretive operator in Banach spaces," Journal of Mathematical Analysis and Applications, vol. 329, no. 1, pp. 415-424, 2007.

[8] P.-E. Maingé, "Viscosity methods for zeroes of accretive operators," Journal of Approximation Theory, vol. 140, no. 2, pp. 127140, 2006.

[9] X. L. Qin, S. Y. Cho, and L. Wang, "Iterative algorithms with errors for zero points of $m$-accretive operators," Fixed Point Theory and Applications, vol. 148, pp. 1-17, 2013.

[10] L.-C. Ceng, S.-Y. Wu, and J.-C. Yao, "New accuracy criteria for modified approximate proximal point algorithms in Hilbert spaces," Taiwanese Journal of Mathematics, vol. 12, no. 7, pp. 1691-1705, 2008.

[11] H.-K. Xu, "Strong convergence of an iterative method for nonexpansive and accretive operators," Journal of Mathematical Analysis and Applications, vol. 314, no. 2, pp. 631-643, 2006.

[12] Y. J. Cho, S. M. Kang, and H. Zhou, "Approximate proximal point algorithms for finding zeroes of maximal monotone operators in Hilbert spaces," Journal of Inequalities and Applications, vol. 2008, Article ID 598191, 10 pages, 2008. 
[13] L.-C. Ceng, A. R. Khan, Q. H. Ansari, and J.-C. Yao, "Strong convergence of composite iterative schemes for zeros of $m$ accretive operators in Banach spaces," Nonlinear Analysis: Theory, Methods \& Applications, vol. 70, no. 5, pp. 1830-1840, 2009.

[14] R. Chen, Y. Liu, and X. Shen, "Iterative approximation of a zero of accretive operator in Banach space," Nonlinear Analysis: Theory, Methods \& Applications, vol. 71, no. 12, pp. e346-e350, 2009.

[15] H. Zegeye and N. Shahzad, "Strong convergence theorems for a common zero for a finite family of $m$-accretive mappings," Nonlinear Analysis: Theory, Methods \& Applications, vol. 66, no. 5, pp. 1161-1169, 2007.

[16] L. Hu and L. Liu, "A new iterative algorithm for common solutions of a finite family of accretive operators," Nonlinear Analysis: Theory, Methods \& Applications, vol. 70, no. 6, pp. 2344-2351, 2009.

[17] Y. Yao, Y. C. Liou, and G. Marino, "Strong convergence of two iterative algorithms for nonexpansive mappings in Hilbert spaces," Fixed Point Theory and Applications, vol. 2009, Article ID 279058, 7 pages, 2009.

[18] F. E. Browder, "Nonexpansive nonlinear operators in a Banach space," Proceedings of the National Academy of Sciences of the United States of America, vol. 54, pp. 1041-1044, 1965.

[19] H.-K. Xu, "Iterative algorithms for nonlinear operators," Journal of the London Mathematical Society, vol. 66, no. 1, pp. 240-256, 2002.

[20] T. Suzuki, "Strong convergence theorems for infinite families of nonexpansive mappings in general Banach spaces," Fixed Point Theory and Applications, vol. 2005, Article ID 685918, 2005.

[21] V. Barbu, Nonlinear Semigroups and Differential Equations in Banach Space, Noordhoff, Leyden, The Netherlands, 1976.

[22] S. Reich, "Approximating fixed points of nonexpansive mappings," Panamerican Mathematical Journal, vol. 4, no. 2, pp. 2328, 1994.

[23] W. Li and H. Zhen, "The applications of theories of accretive operators to nonlinear elliptic boundary value problems in $L^{p_{-}}$ spaces," Nonlinear Analysis: Theory, Methods \& Applications, vol. 46, no. 2, pp. 199-211, 2001.

[24] L. Wei and Z. He, "Existence of solutions to nonlinear Neumann boundary value problems with generalized $p$-Laplacian operator," Computers \& Mathematics with Applications, vol. 56, no. 2, pp. 530-541, 2008.

[25] L. L. Li and Y. T. Guo, The Theory of Sobolev Space, Shanghai Science and Technology Press, Shanghai, China, 1981.

[26] L. Wei and Z. He, "The applications of sums of ranges of accretive operators to nonlinear equations involving the $p$ Laplacian operator," Nonlinear Analysis: Theory, Methods \& Applications, vol. 24, no. 2, pp. 185-193, 1995. 


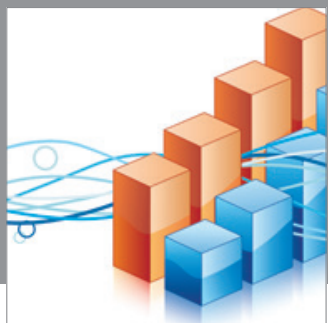

Advances in

Operations Research

mansans

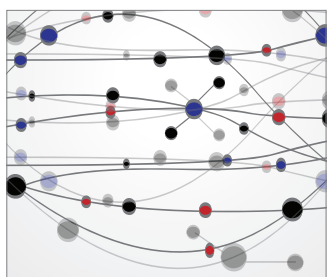

The Scientific World Journal
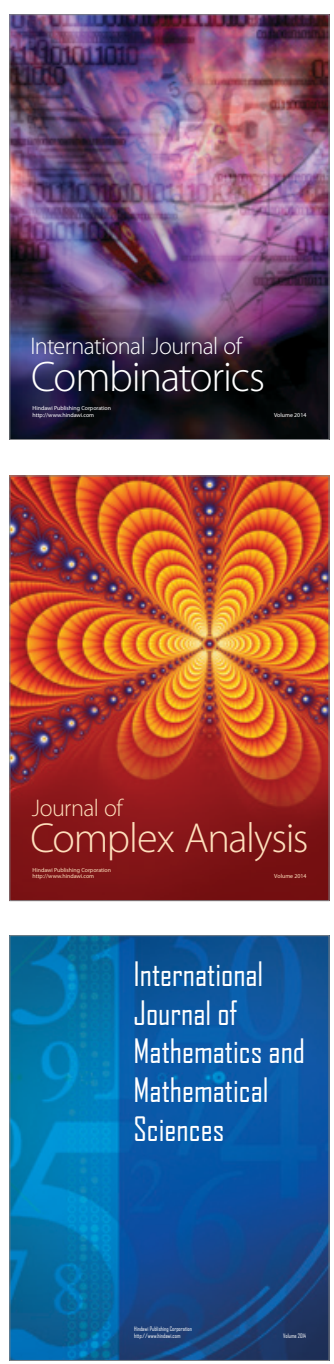
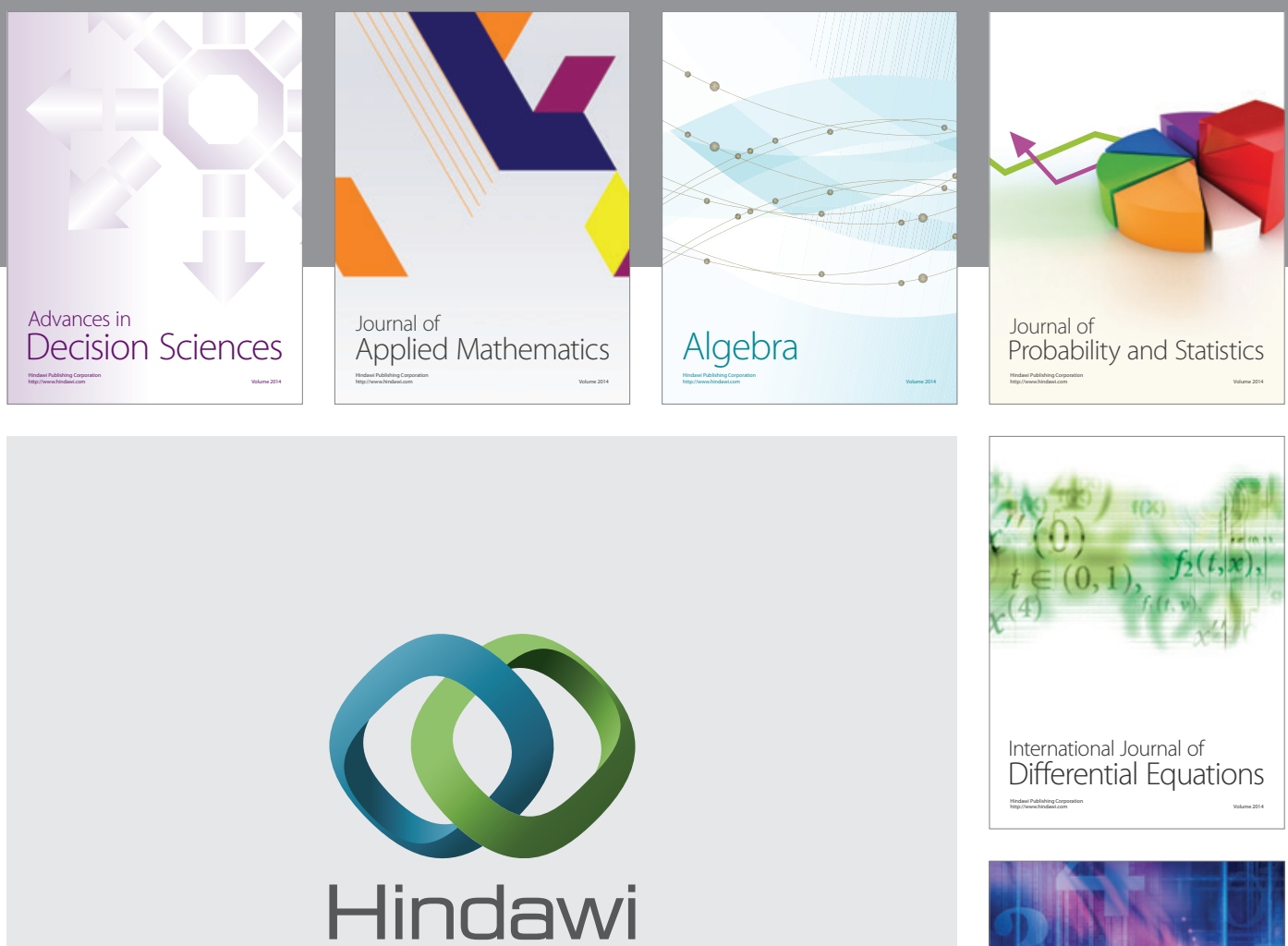

Submit your manuscripts at http://www.hindawi.com
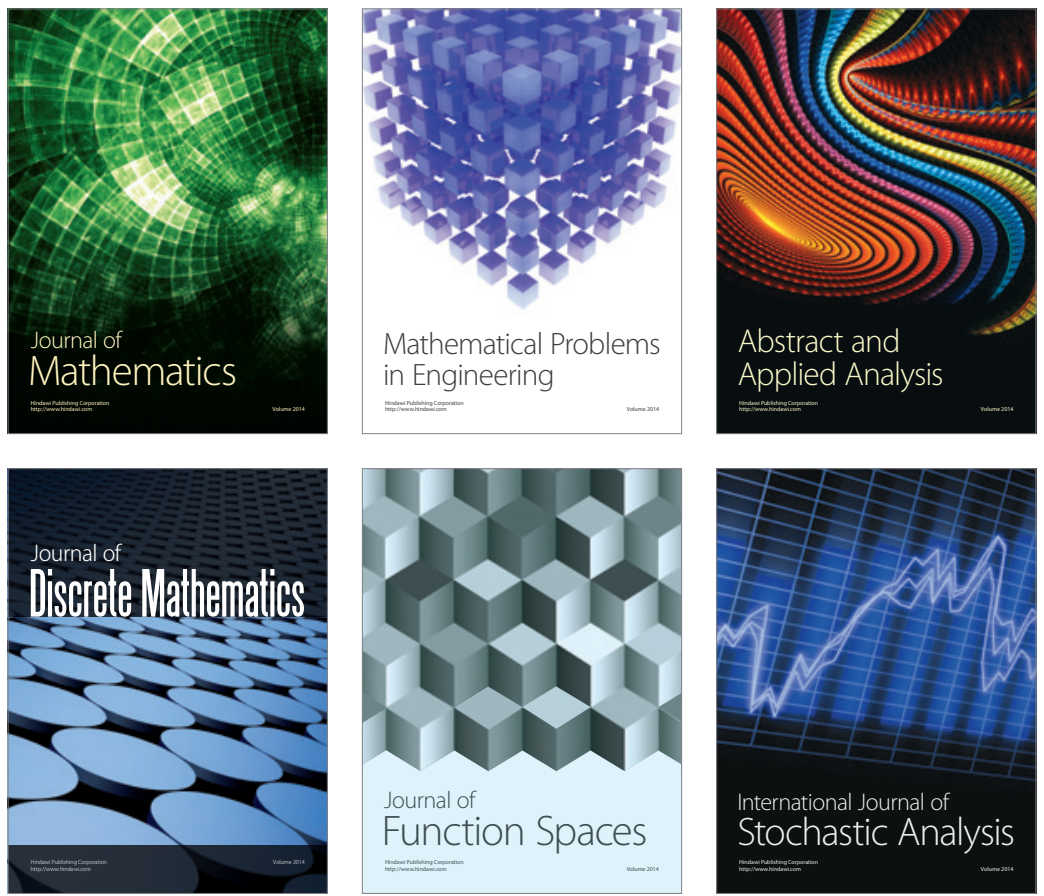

Journal of

Function Spaces

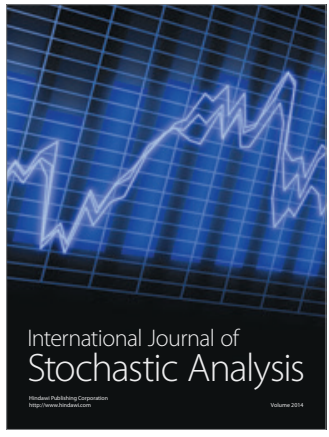

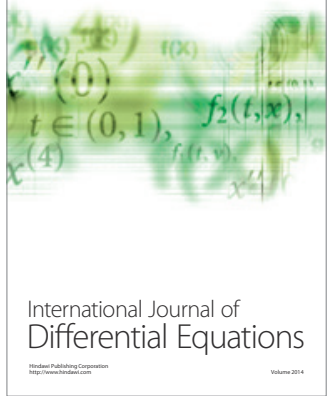
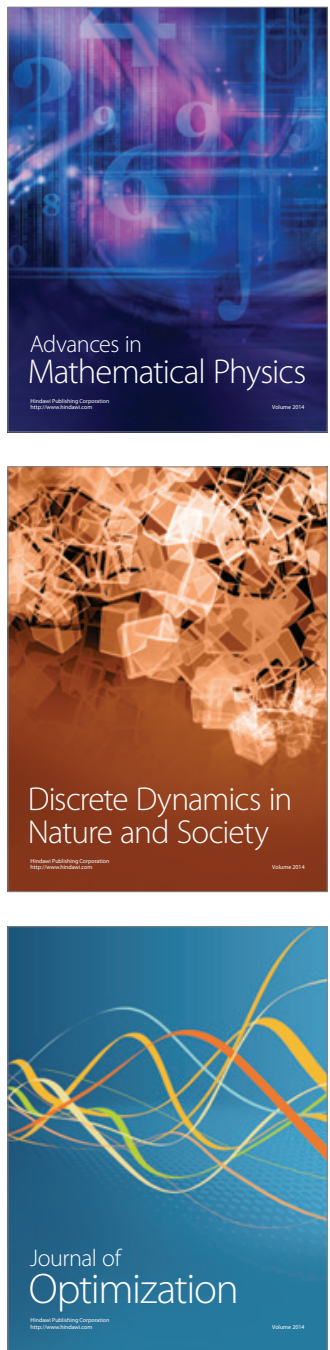